\title{
Automated Labeling of Bronchial Branches in Virtual Bronchoscopy System
}

\author{
Kensaku MORI ${ }^{1}$, Jun-ichi HASEGAWA ${ }^{2}$, Yasuhito SUENAGA ${ }^{1}$, \\ Jun-ichiro TORIWAKI ${ }^{3}$, Hirofumi ANNO $^{4}$, and Kazuhiro KATADA ${ }^{4}$
}

1 Dept. of Computational Science and Engineering, Graduate School of Engineering, Nagoya University, Furo-cho, Chikusa-ku, Nagoya, Aichi, 464-8603 JAPAN \{mori, suenaga\}@nuie.nagoya-u.ac.jp,

WWW home page: http://wwr.toriwaki.nuie.nagoya-u.ac.jp/ mori

${ }^{2}$ School of Computer and Cognitive Sciences, Chukyo University, 101, Tokodachi, Kaizu-cho, Toyota, Aichi, 470-0348 JAPAN hasegaraesccs . chukyo-u. ac.jp

3 Dept. of Information Engineering, Graduate School of Engineering, Nagoya University, Furo-cho, Chikusa-ku, Nagoya, Aichi, 464-8603 JAPAN toriwaki@nuie.nagoya-u.ac.jp

${ }^{4}$ School of Health Sciences, Fujita Health University, Dengakugakubo, Kutsukake-cho, Toyoake, Aichi, 470-1192 JAPAN \{hanno, kkatada\}@fujita-hu.ac.jp

\begin{abstract}
This paper proposes a method for automated labeling of bronchial branches in virtual bronchoscopy system. Anatomical names of bronchial branches are assigned basing upon a set of rules. If it is possible to assign the anatomical names to bronchial branches extracted from $\mathrm{CT}$ images by the computer and is possible to display the name of the currently observed branch on a virtual bronchoscopy image, it will help us to understand the current observing position. The proposed method constructs the knowledge base of anatomical names of bronchial branches. Tree structure of bronchus region is extracted from CT image. Labeling is performed by comparing tree structure information of the extracted bronchus with information of the branches described in the knowledge base. We implemented the proposed method with the virtual bronchoscopy system and obtained satisfactorily result.
\end{abstract}

\section{Introduction}

Rapid progress of three dimensional imaging equipment enables us to acquire very precise 3-D images of human body. However the doctors' load of diagnosing them is increasing. Therefore useful observation methods for those 3-D image are strongly expected. Virtualized endoscope system (virtual endoscopy system) has been developed as one of new observation tools of 3-D images. This system enables us to fly through inside and outside the human body in real time. If it is possible to display the anatomical name of the organ in a view of virtualized endoscope system corresponding to changing viewpoints and view directions, this will help us to know the current observation point. 
Many researchers have reported a various kind of automated extraction methods of the organ from 3-D medical images. However there was no report about automated assignment of anatomical names to the organs extracted from a 3-D image. While some researchers including authors have reported about virtual endoscopy system, no paper has been published for automated display of the anatomical name of the organ in virtual endoscopy system [1-7]. Hoehne and colleagues have constructed the atlas of human body on computer. Anatomical names which are labeled manually are displayed on the rendered image of organ as the atlas [8]. Our method automatically assigns the anatomical name of the bronchus by using the knowledge base of the anatomical name of the bronchus and display the assigned result on the virtual bronchoscopy image corresponding to the viewpoint and view direction for helping the user to understand the current observation point.

This paper describes about a method for automated assignment of anatomical names of bronchial branches which were extracted from 3-D chest X-ray CT images and its application to virtual bronchoscopy system [9] for displaying branch names in the system. In section 2, the outline of the knowledge base of anatomical names of bronchial branches is briefly described. Section 3 shows the whole procedure of automated assignment and the method for displaying the branch name in the virtual bronchoscopy system. Experimental results are shown in Section 4 and brief discussion is added to the results.

\section{Knowledge Base of Anatomical Names of Bronchial Branch}

Bronchus is the organ which has the tree structure. The anatomical name of each branch is assigned basing upon a set of rules. We implement this assignment rule of anatomical name as knowledge base. Assignment of branch names is performed by comparing this knowledge base and the branching structure of bronchus which was extracted from a given CT image.

The algorithm of this name assignment accompanies the knowledge base of anatomical name of bronchial branch described by the frame representation which is one of the well-known methods for knowledge representation (Fig.1). Each frame keeps the knowledge of each branch consisting of the information of tag name, anatomical name, parent branch name, position and direction of branch, and weighted value for each evaluation item.

\section{Processing Procedure}

\subsection{Automated Labeling of The Anatomical Name of Bronchial Branches}

Fig.2 shows the processing procedure of automated labeling of bronchial branches. In the procedure, the bronchus region is extracted from 3-D CT images. Tree structure of bronchus is obtained from the extracted region. Automated labeling is performed, being based on the extracted tree structure of bronchus. 


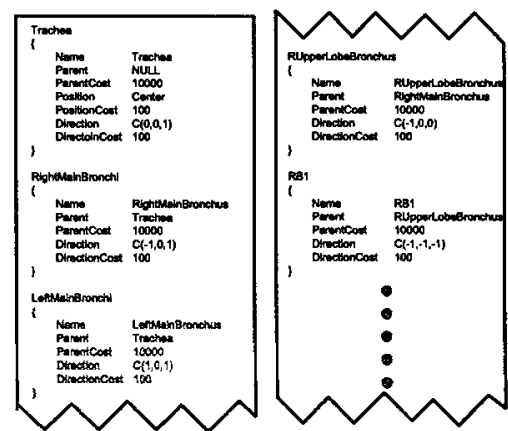

(a)

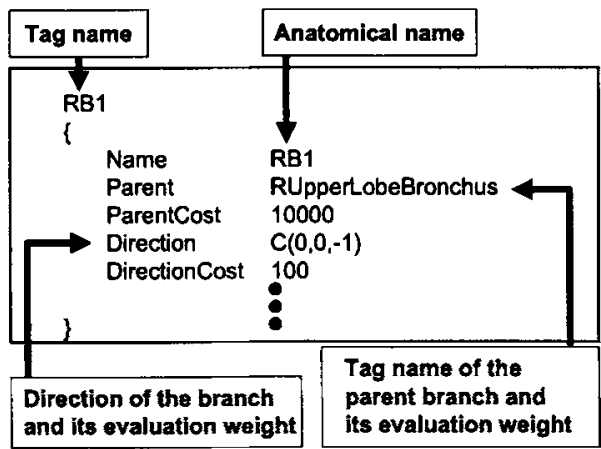

(b)

Fig. 1. An example of the knowledge base represented by frame description method. (a) overview of knowledge base, and (b) knowledge of one branch of the bronchus

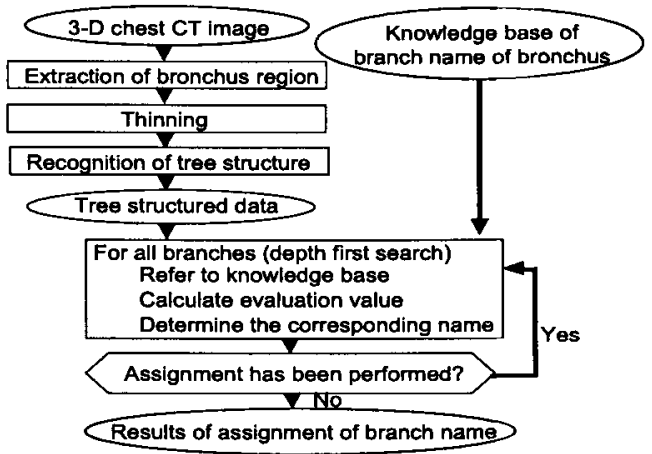

Fig. 2. Automated labeling procedure (automated assignment of anatomical name for bronchial branches)

1) Extraction of Branching Structure of Bronchus (Fig. 3) The bronchus region is extracted from 3-D chest X-ray CT image automatically by employing the method shown in [9]. This method segments the bronchus region by thresholding for CT numbers. The threshold value is automatically adjusted. The medial axis of the bronchus is calculated by using the thinning method [10]. Branching structure of bronchus is extracted from the thinned image [11]. The graph representation is generated also from the thinned result. In the graph, trachea is the root and each branches and branching points of bronchus are represented by arcs and nodes of the graph, respectively. Each arc (branch) has the following information : connecting branching point, direction of the arc, and the pointers to the parent arc and the child arc.

2) Labeling of Anatomical Name for Each Branch This procedure assigns the anatomical name to the bronchial branch by comparing the knowledge base with the structure information extracted in 1). The assignment procedure starts from the trachea and is performed sequentially from the center to the peripheral by the depth first search. The method selects the most appropriate name of a 
bronchial branch, which is described in the knowledge base, for each branch of the bronchial tree. Selection is performed by finding the branches of the knowledge base which has the highest evaluation value. Evaluation value is calculated from the information (such as running direction, parent branch) of each branch which is extracted from a CT image and the information described in the knowledge base. The following shows the detail of the procedure.

step1) The tree structure information of the extracted bronchus and the knowledge base are inputted.

step2) One branch $B_{i}\left(B_{i}\right.$ means the branch $i$ of the bronchus extracted from a given CT image. $B_{i} \in$ \{all branches of the bronchus extracted from CT image\}) is selected from tree structure. This selection is performed sequentially for all branches of the bronchus extracted from CT image by the depth search from trachea.

step3) Evaluation values $E_{i j}$ for $B_{i}$ and $K_{j}$ are calculated ( $K_{j}$ means the branch $j$ described in the $j$-th item of the knowledge base. $K_{j} \in$ all branches described in the knowledge base \}) for all $j$. The evaluation value $E_{i j}$ is calculated by

$$
E_{i j}=w_{d} \frac{\mathbf{d}_{B_{i}} \bullet \mathbf{d}_{K_{j}}}{\left|\mathbf{d}_{B_{i}}\right|\left|\mathbf{d}_{K_{j}}\right|}+w_{p} V
$$

where $\mathbf{d}_{B_{i}}$ represents the running direction of the branch $B_{i}, \mathbf{d}_{K_{j}}$ the running direction of the branch $K_{j} . w_{d}$ and $w_{p}$ are weighted values. $V$ takes $V_{p}$ if the anatomical name of the parent branch of $B_{i}$ is equal to the name of the parent branch of $K_{j}$, or 0 if the name is not equal.

step4) The branch $K_{j \max }$, which has the highest evaluation value for $B_{i}$ and its evaluation value is greater than a given threshold value, is selected from a set of branches described in the knowledge base. Then we assign the anatomical name of $K_{j m a x}$ to the branch $B_{i}$. If we could not find the branch $K_{j \max }$ which satisfies the above stating condition, assignment is not performed for $B_{i}$. If $K_{j \max }$ was already assigned to another branch $B_{l}$, we compare two evaluation values $E_{i j \max }$ (evaluation value for $B_{i}$ and $K_{j \max }$ ) and $E_{l j \max }$ (evaluation value for $B_{l}$ and $K_{j \max }$ ). If $E_{i j \max }>E_{l j \max }$, the branch name of $K_{j \max }$ is assigned to $B_{i}$ and assignment to $B_{l}$ and its child branches are canceled. Otherwise the assignment is not performed.

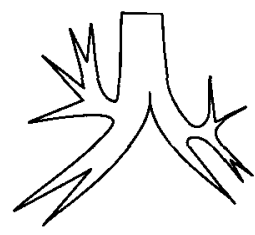

Bronchus region

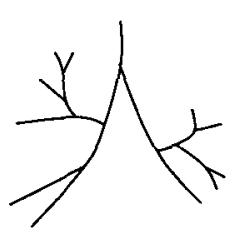

Thinning
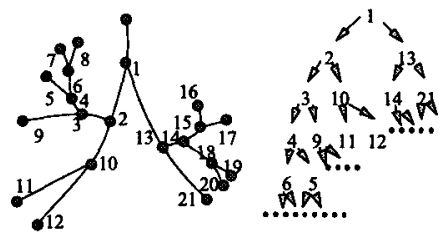

Recognition of Tree structure branches and $\Rightarrow$ of bronchus branching points

Fig. 3. Extraction of the tree structure of bronchus from a 3-D CT image 


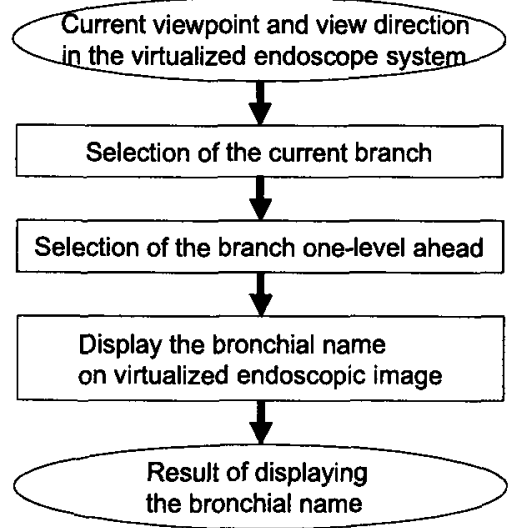

Fig. 4. Processing procedure for automated display of anatomical names of bronchial branches

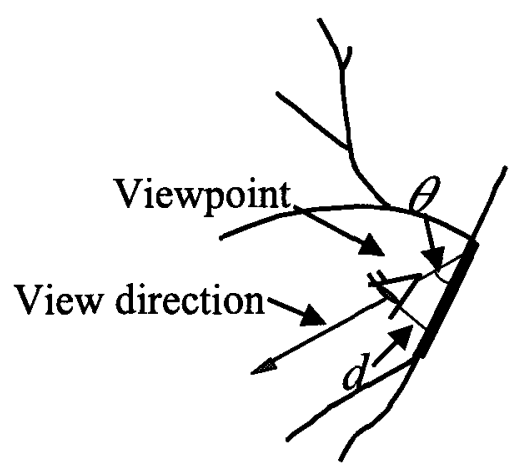

Fig. 5. Selection of the branch corresponding to the current view point

step5) If all branches have been processed, go to step6). Otherwise, go to step2).

step6) If new assignment is performed in the iteration of step2)-step6), go to step2) and start from trachea again. Otherwise, labeling is terminated.

\subsection{Display of The Branch Name in Virtual Bronchoscopy System}

Anatomical names of bronchial branches are displayed in the virtual bronchoscopy system (Fig.4) which we have developed [4,6]. In the virtual bronchoscopy system, bronchus region is extracted from a 3-D chest X-ray CT image. The shape of bronchus is represented by triangle patches. Marching Cubes method [12] was employed here for patch generation and the triangle patches are rendered by gouraud shading, perspective projection and spot light model. The user can specify the viewpoint and the view direction by mouse interactively and the corresponding image is rendered in real time.

The branch corresponding to the current viewpoint is found from the branching structure of the bronchus extracted in 3.11 ). The current branch is determined by calculating the closest branch from the current viewpoint. The current observation direction ("from center to peripheral" or "from peripheral to center") is also found automatically by the angle of the current view direction and the direction of the closest branch (Fig.5). When the observation direction is "from center to peripheral", we select two types of branches for displaying anatomical names, the branch which corresponds to the current viewpoint and the branches which are one level ahead (Fig.6). When the view direction is "from peripheral to center", we select the branch which corresponds to the current view point and the branch which is one level behind. Anatomical names of the selected branches are displayed in the virtual bronchoscopy image by character string in English at the positions corresponding to the selected branches. When the viewpoint and the view direction are changed, the name of branch and its displaying position are also changed. 


\section{Experimental Result and Discussion}

\subsection{Automated Labeling of Bronchial Branches}

We applied the proposed method to eleven cases of real 3-D chest X-ray CT images which were taken by helical CT scanner. Image specifications are follows : image size is 512 (pixels) x 512 (pixels), the number of slices is 60 - 118 (slices), pixel size is $0.63 \mathrm{~mm}$, reconstruction pitch is $1 \mathrm{~mm}$ or $2 \mathrm{~mm}$, width of X-ray beam is $2 \mathrm{~mm}$ or $5 \mathrm{~mm}$. Knowledge base was created manually from textbook of anatomy. As the evaluation items for assignment, we used the parent name and the direction of the branch. Fig. 7(a) displays the outside view of bronchus extracted from CT image. Fig.7 (b) is the thinned results of the extracted bronchus region. Fig.7 (c) shows the tree structure and assignment results of anatomical names. Table 1 shows the results of assignments. The experimental result showed that this methods can assign correct anatomical names to about $90 \%$ of the extracted branches. This methods is considered as one of the method which uses knowledge based processing for medical image processing. This method will be very useful for automated description by anatomical name of the lung cancer extracted automatically [13].

\subsection{Automated Display of Anatomical Name in The Virtual Bronchoscopy System}

Fig. 8 shows the scene of displaying the branch name in the virtual bronchoscopy system. Anatomical names are appropriately selected and automatically displayed on the images rendered by the virtual bronchoscopy system. Frame rate was about 6 frames / second. The computer was Silicon Graphics Octane MXI (cpu :R10000 195MHz, main memory: 512MByte). We confirmed that automatic display of branch name is very useful for understanding the current observation location.

The proposed method enables to display anatomical name on the endoscopic image rendered by the virtual bronchoscopy system. This is one of new diagnosis systems of medical images, which combines the observation method of 3-D medical images and the implementation of the knowledge based processing. Furthermore it is especially useful for education of medical students who are learning branching structure of the bronchus. Authors are collaborating with medical doc-

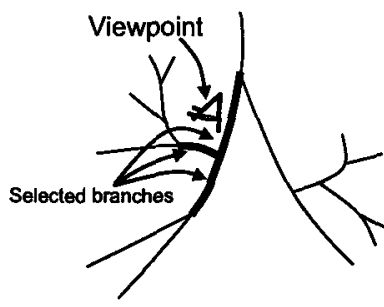

Center to Peripheral

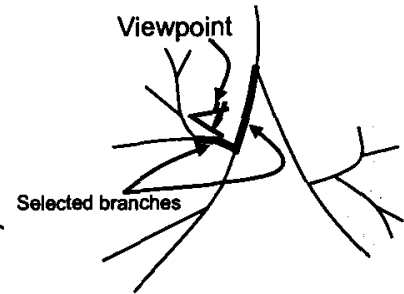

Peripheral to Center

Fig. 6. Selection of the bronchial branches for displaying anatomical name 


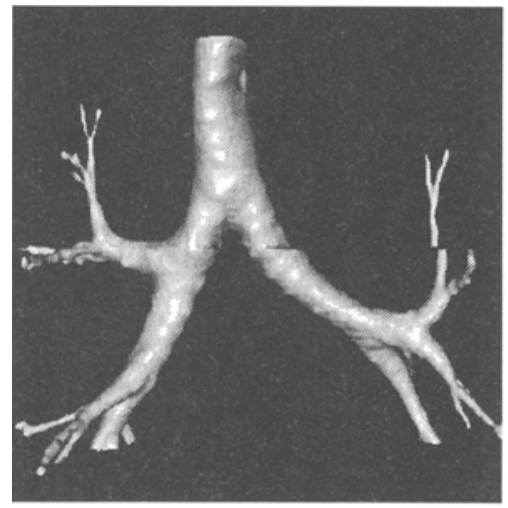

(a)

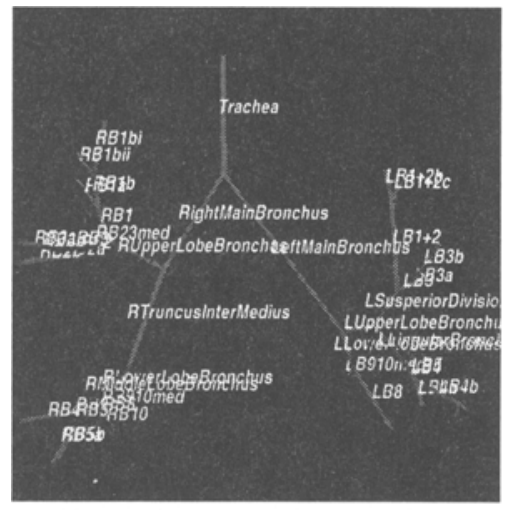

(c)

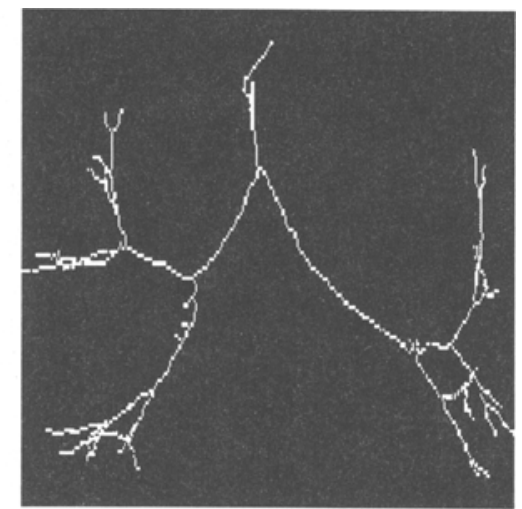

(b)

Table 1. Results of automated assignments of the anatomical name of

\begin{tabular}{ccc}
\multicolumn{3}{c}{ bronchial branches } \\
\hline $\begin{array}{c}\text { The number of } \\
\text { Data No. } \\
\text { branches extracted } \\
\text { from images }\end{array}$ & $\begin{array}{l}\text { The number of branches } \\
\text { assigned by proposed } \\
\text { method successfully }\end{array}$ \\
\hline 001 & 28 & 24 \\
002 & 27 & 23 \\
005 & 9 & 9 \\
006 & 9 & 9 \\
007 & 19 & 19 \\
010 & 19 & 15 \\
014 & 9 & 9 \\
018 & 11 & 11 \\
019 & 11 & 11 \\
021 & 43 & 43 \\
024 & 23 & 7
\end{tabular}

Fig. 7. Results of automated assignment of the bronchial names. (a) the extracted region of the bronchus, (b) the thinned result, (c) automated assignment result of the branch names

tors for application of the proposed method to the education at medical college (Fig.9).

\section{Conclusion}

This paper presented the automated assignment method of anatomical names basing upon the knowledge base for bronchial branches, and its application to the virtual bronchoscopy system. Future work includes evaluation by large number of cases, development of assignment algorithm which can treat variation in the shapes of bronchus among patients efficiently, applications to other organs, and extension of the knowledge base.

\section{Acknowledgment}

Authors thank to Dr. Hiroshi Natori of Sapporo Medical University and Dr. Hirotsugu Takabatake of Hokkaido Keiai-kai Minami Ichi-jyo Hospital for pro- 
viding CT images, useful comments and clinical validation of our system. Also authors thank to our colleagues for useful suggestion and discussion. Parts of this research was supported by the Grant-In-Aid for Scientific Research from the Ministry of Education, the Grant-In-Aid for Cancer Research from the Ministry of Health and Welfare of Japanese Government and Hori Information Promotion Foundation.

\section{References}

1. Vining, D.J., Shitrin, R.Y., Haponik, E.F., et. al. : Virtual Bronchoscopy. Radiology 193 (P, Supplement to Radiology (RSNA Scientific Program)) (1994), 261

2. Mori, K., Hasegawa, J., Toriwaki, J., Anno, H., Katada, K. : A method to extract pipe structured components in three dimensional medical images and simulation of bronchus endoscope image. Proc. of 3-D Image Conf.94 (1994) 269-274

3. Mori, K., Hasegawa, J., Toriwaki, J., Anno, H., Katada, K.: Automated extraction and visualization of bronchus from 3-D CT images of Lung. In: Ayache, N. (ed.) : Computer Vision, Virtual reality and Robotics in Medicine. Lecture Notes in Computer Science, Vol. 905. Springer-Verlag, Berlin Heidelberg New York (1995) $542-548$

4. Mori, K., Hasegawa, J., Toriwaki, J., Anno, H., Katada, K. : Bronchus endoscope simulation system based on three dimensional X-ray CT images (Virtualized Bronchus Endoscope System). Japanese Journal of Medical Electronics and Biological Engineering 33 (1995) 343-351

5. Geiger, B., Kikinis, R. : Simulation of Endoscopy. In: Ayache, N. (ed.) : Computer Vision, Virtual reality and Robotics in Medicine. Lecture Notes in Computer Science, Vol. 905. Springer-Verlag, Berlin Heidelberg New York (1995) 542-548

6. Mori, K., Urano, A., Hasegawa, J., Toriwaki, J., Anno, H., Katada, K. : Virtualized Endoscope System - An Application of Virtual Reality Technology to Diagnostic Aid -. IEICE Transaction of Information and System E79-D (1996) 809-819

7. Ge, Y., Stelts, D.R., Vining, D.J. : 3D Skeleton for Virtual Colonoscopy. In: Hoehne, K.H., Kikinis, R. (eds.) : Visualization in Biomedical Computing. Lecture Notes in Computer Science, Vol. 1131. Springer-Verlag, Berlin Heidelberg New York (1996) 449-454

8. Hoehne, K.H., Pflesser, B., Pommert, A., Riemer, M., Schiemann, T., Schubert, R., Tiede, U. : A 'virtual body' model for surgical education and rehearsal. : IEEE computer 29 (1996) 25-31

9. Mori, K., Urano, A., Hasegawa, J., Toriwaki, J., Anno, H., Katada, K. : Recognition of bronchus in three dimensional X-ray CT images with application to virtualized bronchoscopy system. Proc. of 13th ICPR. Vol.III (1996) 528-532

10. Saito, T., Toriwaki, J., : A sequential thinning algorithm for three dimensional digital pictures using the euclidean distance transformation, Proc. of the 8th Scandinavian Conf. on Image Analysis (1995) 507-516

11. Mori, K., Urano, A., Hasegawa, J., Toriwaki, J., Anno, H., Katada, K. : A fast rendering method using the tree structure of objects in virtualized bronchus endoscope system. In: Hoehne, K.H., Kikinis, R. (eds.) : Visualization in Biomedical Computing. Lecture Notes in Computer Science, Vol. 1131. Springer-Verlag, Berlin Heidelberg New York (1996) 33-42

12. Lorensen, W., Cline, H.E. : Marching Cubes - a high resolution 3D surface construction algorithm. Computer graphics 21 (1987) 163-169

13. Kanazawa, K., Kubo, M., Niki, N., et. al. : Computer aided diagnosis for lung cancer based on helical CT image. Proc. of 13th ICPR. Vol.III (1996) 381-385 


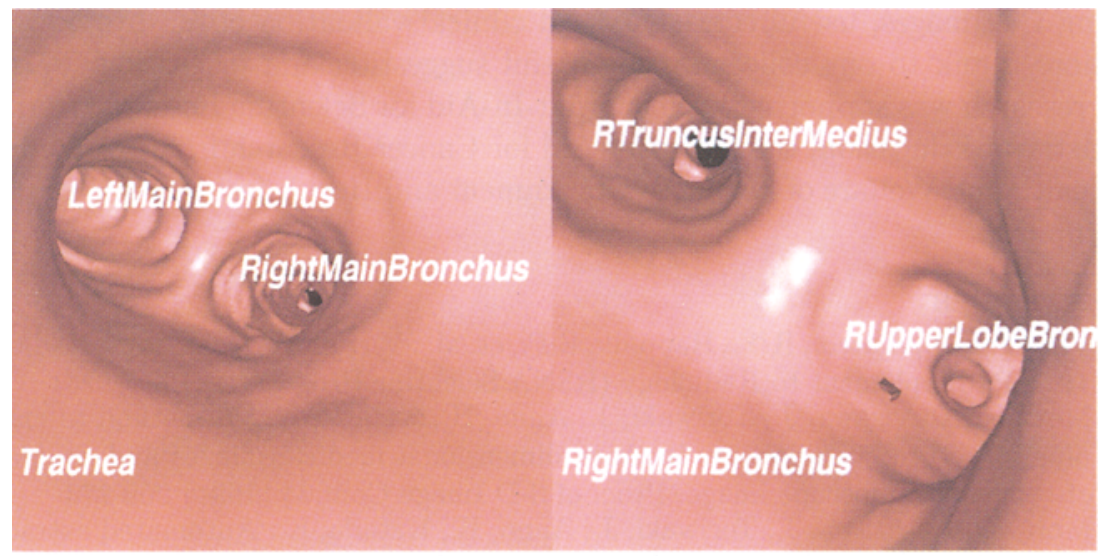

(a)

(b)

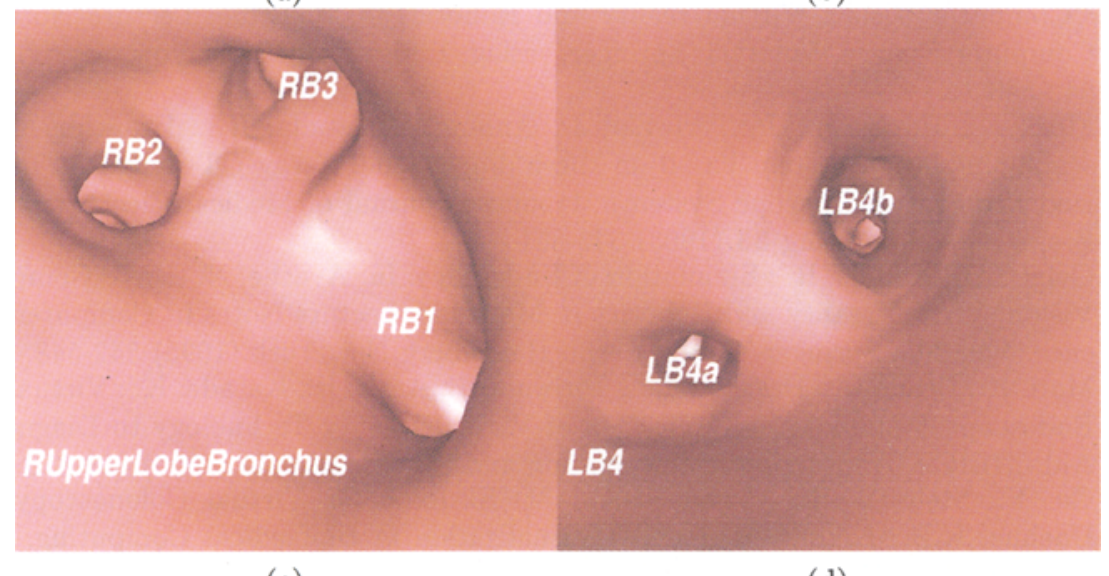

(c)

(d)

Fig. 8. An example of the images of automated display of anatomical names in the virtual bronchoscopy system. (a)-(d) are rendered at different viewpoints and from different view directions.

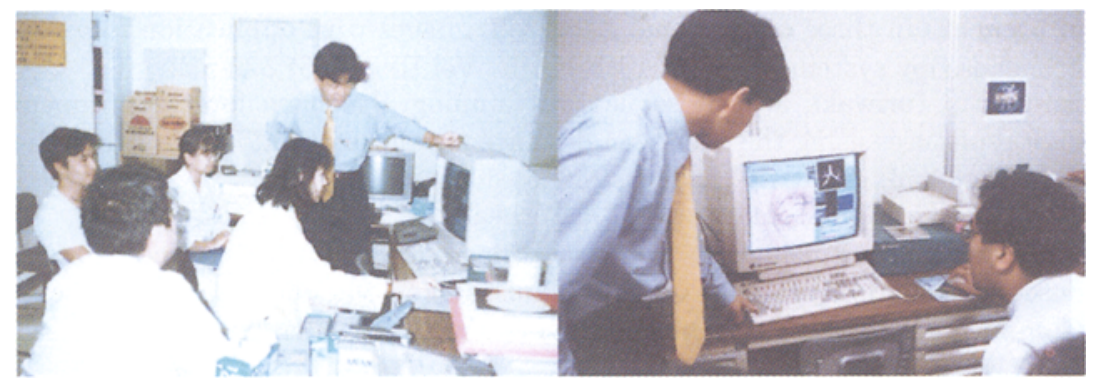

Fig. 9. Scenes of using the proposed system for education of medical students (at Sapporo Medical University, Japan) 